\title{
Ischemic Glomerulopathy
}

National Cancer Institute

\section{Source}

National Cancer Institute. Ischemic Glomerulopathy. NCI Thesaurus. Code C96190.

A renal disorder characterized by glomerular damage due to ischemia. It leads to progressive deterioration of renal function. 